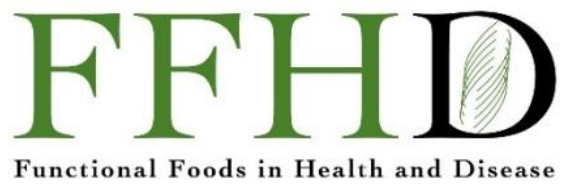

\title{
Curcumin exerts a protective effect against obesity and liver injury induced by an atherogenic diet
}

\author{
María Eugenia Antona ${ }^{1}$, Paula Mariela González ${ }^{2}$, Cecilia Ramos ${ }^{1}$, Joaquín Cabrera ${ }^{2}$, Carolina \\ Olano $^{3}$, Celina Morales ${ }^{4}$, Valeria Zago ${ }^{3}$, Tammy Steimetz ${ }^{5}$, Susana Puntarulo², Silvia María \\ Friedman ${ }^{1 *}$, Elisa Vanesa Macri ${ }^{{ }^{*}}$
}

\begin{abstract}
${ }^{1}$ University of Buenos Aires, Faculty of Dentistry, Department of General and Oral Biochemistry, Marcelo T. de Alvear 2142 12 B (C1122 AAH). Buenos Aires, Argentina; 2University of Buenos Aires, 3Faculty of Pharmacy and Biochemistry, Department of Physical Chemistry, Buenos Aires, Argentina. Institute of Biochemistry and Molecular Medicine (IBIMOL), National Scientific and Technical Research Council (CONICET). Junín 954 (C1113AAD). Buenos Aires, Argentina; ${ }^{3}$ University of Buenos Aires, Institute of Physiopathology and Clinical Biochemistry (INFIBIOC), Buenos Aires, Argentina. The University of Buenos Aires, Faculty of Pharmacy and Biochemistry, Department of Clinical Biochemistry, Laboratory of Lipids and Lipoproteins. Junín 954 (C1113AAD). Buenos Aires, Argentina; ${ }^{4}$ University of Buenos Aires, Faculty of Medicine, Institute of Cardiovascular Pathophysiology (INFICA), J. E. Uriburu 950 (C1114AAD). Buenos Aires, Argentina; ${ }^{5}$ University of Buenos Aires, Faculty of Dentistry, Department of Pathology. Marcelo T. de Alvear 214212 B (C1122 AAH). Buenos Aires, Argentina.
\end{abstract}

*Corresponding Author: Elisa Vanesa Macri, PhD, University of Buenos Aires, Faculty of Dentistry, Department of General and Oral Biochemistry, Marcelo T. de Alvear 214212 B (C1122 AAH). Buenos Aires, Argentina

Submission Date: November 4 $4^{\text {th }}, 2021$; Acceptance Date: December $3^{\text {rd }}, 2021$; Publication Date: December $21^{\text {st }}, 2021$

Please cite this article as: Antona M.E., González P.M., Ramos C., Cabrera J., Olano C., Morales C., Zago V. et al. Curcumin exerts a protective effect against obesity and liver injury induced by an atherogenic diet. Functional Foods in Health and Disease 2021; 11(12): 673-689. DOI: https://www.doi.org/10.31989/ffhd.v11i12.862

\section{ABSTRACT}

Background: Curcumin (Cur) is a natural yellow polyphenol extracted from the turmeric rhizome (Curcuma longa). Cur is known for its potential therapeutic properties as an analgesic, anti-inflammatory, antioxidant, antimicrobial, hepatoprotective, and anti-mutagenic, although some of these biological activities remain unproven. Epidemiological studies have shown a positive relationship between high-fat diets and diet-related chronic diseases. We hypothesized that some adverse effects of consuming atherogenic or high-fat diets (AD) can be ameliorated by Cur supplementation. Using an experimental model of rats, this study investigated the significance of Cur when it is given as a supplement in an AD. 
Methods: Healthy adult Wistar rats were randomly assigned to one of three groups. Controls (C) received a standard diet and experimental rats were fed with $A D$ or $A D+C u r$ for 5 weeks. Cur (100 mg/kg body weight) was given orally daily, plus piperine ( $5 \mathrm{mg} / \mathrm{kg}$ body weight). The effect of Cur supplementation was studied on zoometrics, visceral fat content, serum lipids profile, hepatosteatosis, liver function and oxidative status.

Results: Diets did not alter energy consumption. As compared to the other groups, AD+Cur group showed a lower total visceral fat content, percentage of perirenal, mesenteric, and pelvic fat, and body weight gain $(P<0.05)$. Serum total cholesterol $(P<0.0001)$, non-HDL-C $(P<0.0001)$ levels were significantly higher in AD groups as compared with $C$. Serum triglycerides and HDL-C levels remained similar among groups ( $P>0.05)$. AD induced a liver injury with macrovesicular steatosis and portal inflammation. $A D+C u r$ rats presented microvesicular steatosis with no inflammation, achieving the lowest level of alanine aminotransferase $(A L T ; P<0.0001)$ and reductions of aspartate aminotransferase $(A S T ; P<0.0001)$. Liver homogenates from $A D+C u r$ showed that Cur supplementation reduced the dichlorofluorescein diacetate (DCFH-DA) oxidation rate induced by AD by $25 \%$ and deferoxamine and superoxide dismutase inhibited DCFH-DA.

Conclusion: Cur as a dietary supplement showed a protective effect against obesity and inflammation, but its cardioprotective ability remained unproved. Cur may develop as a promising therapeutic agent for liver diseases induced by oxidative stress. This study provides supporting evidence to confirm the beneficial effects of curcumin from the point of view of functional food science.

Keywords: curcumin, liver injury, ROS, atherogenic diet, visceral fat, obesity

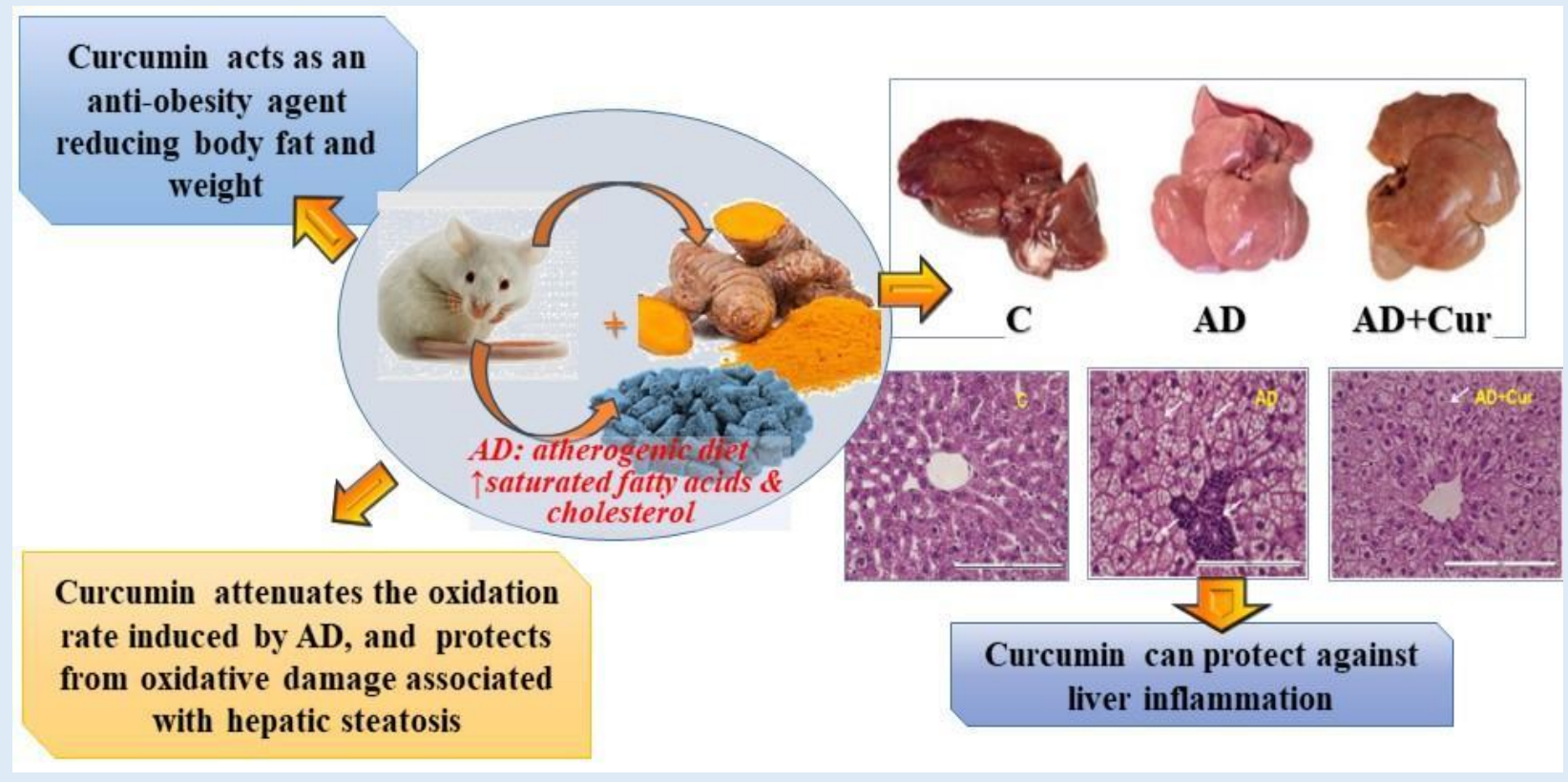

CFFC 2021. This is an Open Access article distributed under the terms of the Creative Commons Attribution 4.0 License (http://creativecommons.org/licenses/by/4.0) 


\section{INTRODUCTION}

In the past few years, the increased sedentary lifestyle and consumption of food rich in saturated fats and cholesterol have exposed the population of developed countries to emerging health problems [1]. The steadily increasing incidence of obesity, cardiovascular disease (CVD) linked to the presence of hyperlipidemia, hypertension, diabetes mellitus, cancers, digestive diseases, and some skeletal, kidney, and liver diseases [23] can be mentioned among these emerging health problems. In the Americas, obesity has reached epidemic proportions, and prevalence is higher in women [4]. In addition, it was recently shown that the origin of these diet-related chronic diseases (DRCD) is multifactorial and may result from different deregulated metabolic parameters, including antioxidant status, acid-base imbalance, increased inflammatory status, and impaired carbohydrate/lipid/one-carbon metabolism [3].

Atherogenic and high-fat diets (AD) in animals tend to develop DRCD and liver damage, similar to the phenotype observed in humans with non-alcoholic fatty liver disease (NAFLD) [5]. NAFLD is characterized by fat accumulation in hepatocytes in the absence of alcohol intake. It is estimated that $25 \%$ of the world's population has NAFLD [6]. In South America, this figure reaches $30.5 \%$ [7]. Non-alcoholic steatohepatitis develops when physiological adaptive mechanisms of the liver are overwhelmed by the excessive influx of triglycerides (TG). This leads to lipotoxicity, inflammation, reactive oxygen species (ROS) formation, and hepatocellular dysfunction [8].

Oxidative stress is generated when ROS formation is not compensated by the antioxidant defense capability or redox signaling is disrupted, affecting cell functionality [9]. This metabolic alteration is an important factor in the pathogenesis of hepatocyte dysfunction [10]. Furthermore, an AD clearly affects the hepatocyte energy metabolism leading to depressed b-oxidation and increased mitochondrial ROS production [11]. Undoubtedly, ROS play a crucial role in the development of numerous chronic liver diseases and stimulate their progression [12].

Natural products represent a source of discovery of new basis for therapeutics guides that can be used in treating different types of DRCD. Patients use herbal medicines due to their wide availability, low toxicity, pharmacological activity, chemical diversity and low side effects compared to synthetic drugs [13]. Curcumin [1,7bis(4-hydroxy-3-methoxyphenyl)-1,6-heptadiene3,5dione] (Cur) is a natural yellow polyphenol extracted from the turmeric rhizome (Curcuma longa), a plant that grows in tropical and subtropical regions throughout the world, which is extensively used for food preparation in many Asian countries [14]. Cur was incorporated into cooking and therapeutic preparations throughout the centuries in different parts of the world and was used as a combined herbal medicine in the treatment of various diseases due to its antioxidant, anti-inflammatory, antimutagenic, antimicrobial and anticancer properties [15-16]. In vivo and in vitro studies revealed that Cur exerts anti-obesity and anti-inflammatory effects by decreasing adiposity, lipid storage, and increasing the oxidation of fatty acids [17].

We hypothesized that Cur can ameliorate some adverse effects of consuming $A D$ by modulating plasma lipid levels, decreasing body fat mass and preventing liver injury. In an experimental model of rats, this study tested the hypothesis investigating the significance of Cur when it is given as a supplement in an AD. The effect of Cur supplementation was studied on zoometrics, visceral fat content, serum lipids profile, hepatosteatosis, liver function and oxidative status. 


\section{METHODS}

Animals: Healthy adult female Wistar rats $(n=21)$ with an initial body weight of $200 \pm 20$ g (aged: $44 \pm 2$ days), were used in this trial. The animals were obtained from the laboratory of the Department of Biochemistry, Faculty of Dentistry, University of Buenos Aires, Argentina. Animals were housed in galvanized cages with meshed floors to maintain hygienic conditions and avoid coprophagy. Rats were kept in individual cages and exposed to a 12-h light/dark cycle throughout the study. The room temperature was maintained at $21 \pm 1^{\circ} \mathrm{C}$ with a humidity of $50-60 \%$.

Ethics: This study was approved by the University of Buenos Aires, Argentina by the Ethical Commission of the School of Dentistry, under Protocol (UBACYT 20020150200013BA, Res CD 398/151). The animals were maintained in accordance with the Guide of the National Academy of Science Animal Welfare Regulations [18].
The study was a part of the doctoral thesis of the lead author of the paper (MEA).

Diets: The composition of the diets is shown in Table 1. The control (C) diet was a standard diet (Purina chow; Gilardoni SA, Buenos Aires, Argentina). The AD used butter as the main source of fat ( $80 \%$ fat) and contained a $15.0 \%$ fat/100 g diet; it was rich in saturated fatty acids and cholesterol (1.51 g/100 g diet). All diets had similar grain sizes. The ingredients of the two diets were milled, mixed and the homogenized material was transferred to the press. The diets were manually cut to achieve a size suitable for rodents, similar to the commercial stock diet. Diets were prepared every 2 days and stored at $-4^{\circ} \mathrm{C}$ until fed. Fresh diets were offered daily and food containers were cleaned before being refilled. Food cups were refilled once a day, and food consumption was measured with a Mettler scale PC 4000 (accuracy $\pm 1 \mathrm{mg}$ ). Daily food intake was recorded as kcal per $100 \mathrm{~g}$ of body weight per day (kcal/100 g BW/day).

Table 1. Composition of standard and experimental diet

\section{Ingredients (g/100 g)}

\section{Energy}

kcal

$\mathrm{kJ}$

\section{Carbohydrates}

Protein (mix of corn, wheat, soybean, fish, and meat flour)

Total Fat (acid hydrolysis) (mix of corn oil, fish oil, and butter)

Saturated fatty acids

Monounsaturated fatty acids

Polyunsaturated fatty acids n-6

Polyunsaturated fatty acids n-3

Cholesterol

Fiber Crude

Ash

Vitamin mixture

Water

\section{C}

AD

\begin{tabular}{l|l}
270 & 337 \\
1130 & 1412 \\
40.00 & 34.00 \\
19.00 & 16.00 \\
4.00 & 15.00 \\
1.65 & 9.00 \\
1.20 & 4.20 \\
1.12 & 1.60 \\
0.03 & 0.20 \\
0.01 & 1.51 \\
5.13 & 4.50 \\
8.06 & 7.10 \\
0.80 & 0.80 \\
23.00 & 21.00 \\
\hline
\end{tabular}

C: control standard diet consisted of commercially available pellets (Purina chow). AD: atherogenic diet, rich in saturated fat and cholesterol. 
Experimental design: Firstly, Rats $(n=21)$ were weighed and numbered according to their body weight. Then, they were assigned into three groups ( $n=7 /$ group): C, control group, was given access to a standard diet; group fed with $A D$ ( $A D$ group), and $A D+$ Cur group were given an $A D$ plus Cur to study the effects of Cur in an $A D-$ fed condition. The composition of the experimental diets is described in the section Diets. The dose of $100 \mathrm{mg} / \mathrm{kg}$ body weight (BW)/day of Cur [from Cúrcuma longa (Tumeric), as powder. Sigma Aldrich S.R.L] was calculated according to the bibliography [19] considering the absence of adverse toxicological effects. Cur was coadministered orally with piperine $(5 \mathrm{mg} / \mathrm{kg}$ BW/day; Sigma Aldrich S.R.L) to enhance Cur's bioavailability [20]. Cur and piperine were dissolved in $0.1 \%$ carboxymethyl cellulose (CMC) and given through oral gavage to the animals. Groups $C$ and $A D$ received a daily oral administration of the same volume of the CMC alone.

Throughout the 5 weeks of the experimental period, the animals accessed food and water ad libitum. After 5 weeks of intervention, food and water were removed at the end of the dark period (7:00 AM). After $4 \mathrm{~h}$ of fasting, animals were euthanized with an intraperitoneal injection of sodium Thiopental ( $4 \mathrm{mg} / 100 \mathrm{~g}$ BW; ScottCassara. Buenos Aires. Argentina). The total carcass was weighed. Blood samples were obtained by cardiac puncture and rapidly centrifuged at $1500 \mathrm{~g}$ for $15 \mathrm{~min}$. Serum samples were stored at $-20^{\circ} \mathrm{C}$ until biochemical assays were performed. The liver was weighted, one piece was kept at $-70^{\circ} \mathrm{C}$ (for biochemical assays) and another piece was placed in $10 \%$ of formalin for histological evaluation.

Biochemical determinations, hepatic index $(\mathrm{HI})$, histology, and body fat content- distribution were performed in all rats.

Zoometry: Total BW was measured weekly after a fasting period of 2-4 $\mathrm{h}$, which allowed enough time to determine zoometric parameters and others [21]. A Mettler PC 4000 scale (accuracy $60.001 \mathrm{~g}$ ) was used to measure BW.
Hepatic index determination: The liver was removed immediately after euthanasia to avoid dehydration and was weighed with an electronic analytical scale. The data was used to calculate the hepatic index (HI). The liver weight of each rat was normalized to the percentage of the total BW to minimize the individual differences in body size. This index was expressed as liver weight/total BW $\times 100$ (HI \%) [22]. The $\mathrm{HI}$ was used to determine enlargement of the liver (hepatomegaly).

Measurement of visceral fat: Fat mass was measured by an analytical balance (Mettler Toledo) and assessed by weighing the perirenal, mesenteric, and pelvic white adipose tissue [23]. The sum of these fat tissues was considered visceral fat. The data were expressed as a percent of the total BW.

Measurement of liver fat content: Hepatic lipid content was determined by a Folch extraction and evaporation to dryness followed by a gravimetric measurement [24]. Pieces of liver were weighed and homogenized with 30 vol of chloroform: methanol (2:1). After standing overnight at room temperature, the homogenate was filtered, and a partition was performed in a separatory funnel by adding 0.2 vol of $0.05 \mathrm{~N}$ aqueous $\mathrm{NaCl}$ solution. When the two phases were completely separated, the lower fraction that contained the lipids dissolved in it was collected. Anhydrite $\mathrm{CaCl} 2$ was added to remove water vestiges and once again filtered to eliminate the salt. The filtered organic phase was dried in a rotavapor at $45^{\circ} \mathrm{C}$. The residue was weighed, and the lipid content was expressed as weight/weight.

Histological evaluation: After the liver was removed, one piece was placed in $10 \%$ of formalin for histological evaluation. Liver histological examination by light microscopy was performed in a blinded manner. Hepatic tissue samples were fixed overnight, at room temperature in a $10 \%$ formalin buffer, $\mathrm{pH} 7$ with $0.1 \mathrm{M}$ phosphate-buffered saline solution. Samples were dehydrated in ethanol, embedded in paraffin wax, and 
cut with a microtome. The resulting 5 micro-sections were stained with hematoxylin and eosin reagent for steatosis evaluation. The steatosis pattern was expressed with the presence of macro and microvesicular fat deposits and portal inflammation [25-26]. The histological features were assessed under low magnification (100x and 200x, respectively).

Biochemical determinations: Serum levels of aspartate aminotransferase (AST) and alanine aminotransferase (ALT) activities were quantified by the UV-Kinetic (IFCC) method (Autopack, Wiener Diagnostics) in a CB350i autoanalyzer (Wiener Lab Rosario, Argentina).

Total serum cholesterol ( $\mathrm{T}-\mathrm{C} ; \mathrm{mg} / \mathrm{dL}$ of serum), highdensity lipoprotein-cholesterol (HDL-C; $\mathrm{mg} / \mathrm{dL}$ of serum), and triglycerides (TG; $\mathrm{mg} / \mathrm{dL}$ of serum) were determined by standardized methods (Roche Diagnostics $\mathrm{GmbH}$, Mannheim, Germany) in a Hitachi 917 autoanalyzer (Hitachi, Tokyo, Japan).

Serum non-HDL cholesterol (non-HDL-C mg/dL), a set of atherogenic lipoproteins rich in apo B, was calculated as the difference between the T-C and HDL-C, given the loss of sensitivity of this parameter, which is usually significantly lower than serum HDL-C levels in rats as compared to humans [27].

Determination of oxidative stress indicators: Liver samples from each rat were homogenized $(1: 5 \mathrm{w} / \mathrm{v})$ in a $100 \mathrm{mM}$ Tris-HCl buffer, pH 7.75 with 2 mM EDTA and $5 \mathrm{mM} \mathrm{MgCl}_{2}$ buffer [28]. Measurements were conducted according to Amado et al. [29] and Viarengo et al. [30] with modifications. Briefly, the homogenates were centrifuged at $4^{\circ} \mathrm{C}$ for $20 \mathrm{~min}$ at $10,000 \mathrm{~g}$ and the supernatants were used for the assay. The reaction was followed in a $30 \mathrm{mM}$ HEPES buffer, pH 7.2 with $200 \mathrm{mM}$ $\mathrm{KCl}$ and $1 \mathrm{mM} \mathrm{MgCl}_{2}$. The fluorescent probe $2^{\prime}, 7^{\prime}$ dichlorofluorescein diacetate (DCFH-DA) was added to the buffer at a final concentration of $40 \mu \mathrm{M}$. Then, after the addition of 5 to $15 \mu$ l of the sample, the reaction mixture was incubated at $35^{\circ} \mathrm{C}$ for $15 \mathrm{~min}$. The fluorescent compound DCF, generated by the radical- dependent oxidation of the probe, was detected spectrofluorometrically at $\lambda_{\mathrm{exc}}=488 \mathrm{~nm}$ and

$\lambda_{\text {em }}=525 \mathrm{~nm} \quad(\mathrm{~F}-3010$ Hitachi $)$. The results were expressed as arbitrary units/min $\mathrm{mg}$ of protein ( $\mathrm{AU} / \mathrm{min}$ $\mathrm{mg}$ prot). Protein content was calculated according to Lowry et al. [31] to standardize the quality of the sample.

The presence of different reactive species was tested by adding the enzymes superoxide dismutase (SOD, $300 \mathrm{U} / \mathrm{ml}$ ) and catalase (CAT, $500 \mathrm{U} / \mathrm{ml}$ ), dimethylsulfoxide (DMSO, $50 \mathrm{mM}$ ), glutathione (GSH, 5 $\mathrm{mM})$, and deferoxamine (DF, $50 \mu \mathrm{M})$. Liver homogenates were incubated with DCFH-DA in the presence of these different scavengers for $15 \mathrm{~min}$ at $35^{\circ} \mathrm{C}$. Then, the oxidation rate of the probe was performed as previously described.

Statistical analyses: To determine the sample size, a program provided by Harvard University (USA) was used (software available at:

http://hedwig.mgh.harvard.edu/sample_size/size.html). The sample size for two groups was calculated using input parameters as follows: $\alpha$ error probability of 0.05 , power probability of 0.08 , and 50 units difference between the means based on previously determined serum T-C levels $(60 \pm 10 \mathrm{mg} / \mathrm{dl})$. As a result, a total sample size of $>6$ rats per group was obtained. The results were expressed as mean values with their standard deviations (SD).

One-way analysis of variance (ANOVA) was used to compare data among groups. When a statistically significant difference was encountered, a StudentNewman-Keul's test was performed. Liver oxidative stress was presented as a percentage of inhibition of each scavenger compared to its basal group. Differences to the basal group were determined with Dunnett's multiple comparisons test. Histological information was analyzed using $\mathrm{Chi}^{2}$ test. In all analyses, Bartlett's test for homogeneous variances was performed. Significance was set at the $p<0.05$ level.

The Statistical Product and Service Solutions for Windows 23 (SPSS, Inc., Chicago, IL) were used for the statistical analyses. 


\section{RESULTS}

BW gain, visceral fat, and food intake: The BW gains

throughout the experimental period, as well as the daily caloric intake of the three groups, are shown in Table 2. At the beginning of the study, the mean body weight was $200 \pm 20 \mathrm{~g}$ for all groups. After 5 weeks of feeding, BW gain was significantly higher in the AD group as compared with $C$ and $A D+C u r$ groups $(P<0.05)$. The $B W$ gain of the
$A D+C$ ur group was similar to the $C$ group $(P>0.05)$. Rats fed a $C$ diet or $A D$ (containing only $A D$ or $A D+C u r$ ) did not show significant differences in energy consumption, despite some differences in energy content, fat content, and saturated fatty acids among the diets. Despite the similar consumption of the diets, supplementing the $A D$ diet with Cur attenuated the BW gain significantly in $A D+$ Cur group.

Table 2. Body and food consumption parameters and liver index in rats

\begin{tabular}{|c|c|c|c|c|}
\hline Parameters & C & AD & AD+Cur & $\boldsymbol{P}$ \\
\hline BW gain*(g) & $81 \pm 11 a$ & $113 \pm 12 b$ & $88 \pm 21 a$ & $<0.05$ \\
\hline Daily calorie intake (Kcal/100 g rat/day) & $27 \pm 2 a$ & $30 \pm 3 a$ & $27 \pm 4 a$ & $>0.05$ \\
\hline $\mathrm{HI}(\mathrm{g} / 100 \mathrm{~g} \mathrm{BW})$ & $4.0 \pm 0.3 a$ & $5.9 \pm 0.2 b$ & $6.1 \pm 0.6 b$ & $<0.001$ \\
\hline
\end{tabular}

C: control group (standard diet); AD: atherogenic diet group (saturated fatty acids and cholesterol diet); $A D+C u r:$ atherogenic diet and curcumin group (saturated fatty acids, cholesterol diet, and oral curcumin). *BW: body weight gain throughout the experimental period. HI: means hepatic index (liver weight/total BW x 100). Different letters indicate statistically significant differences between groups. Oneway ANOVA followed by SNK post hoc was used. Data are expressed as the mean \pm standard deviation of the mean.

Table 3. Effect of administration of Cur on the distribution of visceral fat in AD-fed condition

\begin{tabular}{|l|l|l|l|l|}
\hline Visceral fat & C & AD & AD+Cur & $P$ \\
\hline Perirenal (g/100 g BW) & $1.1 \pm 0.3 \mathrm{a}$ & $1.8 \pm 0.6 \mathrm{~b}$ & $1.0 \pm 0.4 \mathrm{a}$ & $<0.05$ \\
\hline Mesenteric $(\mathrm{g} / 100 \mathrm{~g} \mathrm{BW})$ & $0.26 \pm 0.05 \mathrm{a}$ & $0.34 \pm 0.08 \mathrm{~b}$ & $0.21 \pm 0.03 \mathrm{a}$ & $<0.01$ \\
\hline Pelvic (g/100 g BW) & $1.5 \pm 0.4 \mathrm{a}$ & $2.2 \pm 0.6 \mathrm{~b}$ & $1.3 \pm 0.4 \mathrm{a}$ & $<0.05$ \\
\hline Total visceral fat mass (g/100 g BW) & $2.9 \pm 0.8 \mathrm{a}$ & $4 \pm 1 \mathrm{~b}$ & $2.5 \pm 0.8 \mathrm{a}$ & $<0.05$ \\
\hline
\end{tabular}

Visceral fat weight per $100 \mathrm{~g}$ body weight (BW) of rats. C: control group (standard diet); AD: atherogenic diet group (saturated fatty acids and cholesterol diet); $A D+C u r$ : atherogenic diet and curcumin group (saturated fatty acids, cholesterol diet, and oral curcumin). Different letters indicate statistically significant differences between groups. ANOVA+ Student-Newman-Keul's test (mean \pm SD).

Total visceral fat content and distribution are shown in Table 3. The percentage of total visceral fat and distribution differed among the rats fed with the different diets. Rats consuming an AD diet showed the highest total visceral fat content as compared to $C$ and AD+Cur groups $(P<0.05)$. Furthermore, the percentage of perirenal, mesenteric, and pelvic fat showed the highest values for the $A D$ group; the differences were significant as compared with other groups. Total visceral fat content, $\mathrm{t}$ as well as visceral fat distribution, remained similar within the $C$ and the $A D+C u r$ groups $(P>0.05)$. Cur supplementation in the $A D+C u r$ group significantly reduced the visceral fat; overall, the differential distribution of fat was also affected by the Cur intake. 
Serum lipid profile: The serum lipids of the three groups of rats are shown in Figure 1. The rats fed the $A D$ diet $(A D$ or $A D+C u r)$ showed the highest serum T-C $(P<0.0001)$ and non-HDL-C $(P<0.0001)$ levels; whereas $A D, A D+C u r$, and $\mathrm{C}$ groups attained similar serum concentrations of TG and HDL-C ( $P=0.54$ and $P=0.81$, respectively). Cur supplementation did not induce changes in serum lipid profile when compared to the AD group.

\section{Liver function, histopathology, and oxidative stress:}

The effects of Cur supplementation on liver morphology, fat content, enzymes and histology are presented in Figures 2 and 3. Hepatomegaly was induced by consuming the $A D$. The liver morphologic evaluation indicated qualitative differences among the three groups (Figure 2A). In the AD group, livers were grossly enlarged and became dull pale, slightly soft, friable to the touch and swollen; findings that were compatible with findings consistent with severe fatty liver-like disease; whereas those from rats fed $A D+C$ ur were between sharp red and dull pale. Meanwhile, in group $C$, livers were sharp red and characterized by being soft, flexible, and small in volume.

$A D$ diet induced hepatic steatosis in rats fed $A D$ or $A D+C u r$ as compared to those fed the $C$ diet $(P<0.05$; Figure 2B). In fact, the higher liver fat content in rats fed $\mathrm{AD}$ or $\mathrm{AD}+\mathrm{Cur}$ was consistent with the $\mathrm{HI}(P<0.001$; Table 2).

The increased serum activities of AST and ALT enzymes $(P<0.0001$; Figure 2C, D) denoted liver damage. Rats fed with a high fat and high cholesterol diet provoked the highest levels of both enzymes $(P<0.0001)$; in contrast, the $A D+C u r$ group achieved the lowest levels of $\operatorname{ALT}(P<0.0001)$ and a significant reduction of AST levels $(P<0.01)$ in comparison to those of group $C$.

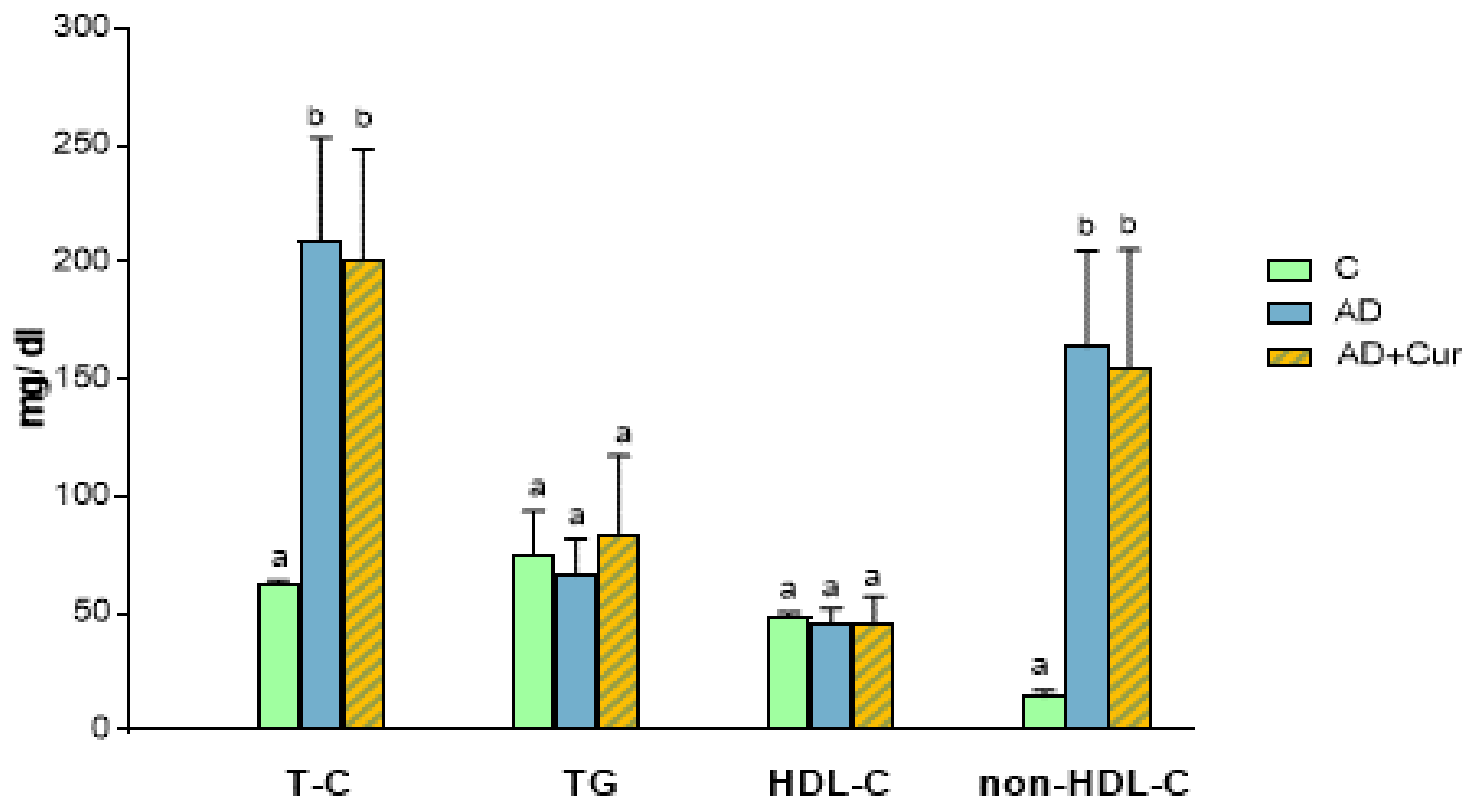

Figure 1. Supplementation with curcumin did not induce changes in serum lipid profile when compared to rats fed an atherogenic diet. Rats were assigned into three groups: $C$, control group, was given access to a standard diet; AD group, fed with atherogenic diet (saturated fatty acids and cholesterol diet) and AD+Cur group (atherogenic diet and curcumin, saturated fatty acids, and cholesterol diet plus oral curcumin). These diets were assigned for five weeks. Serum Total cholesterol (T-C), Triglycerides (TG), High-density lipoprotein (HDL-C), and non-HDL-C (non-HDL-C) were determined. Data are mean values, with standard deviations. Data were analyzed by using ANOVA and Student-Newman-Keul's test $(P<0.05)$. 
(A)

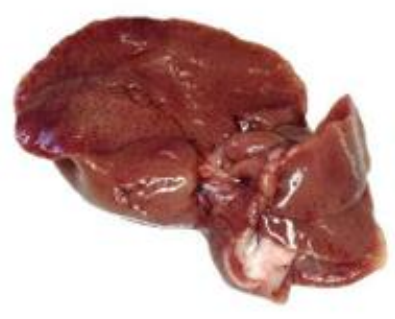

C

(B)

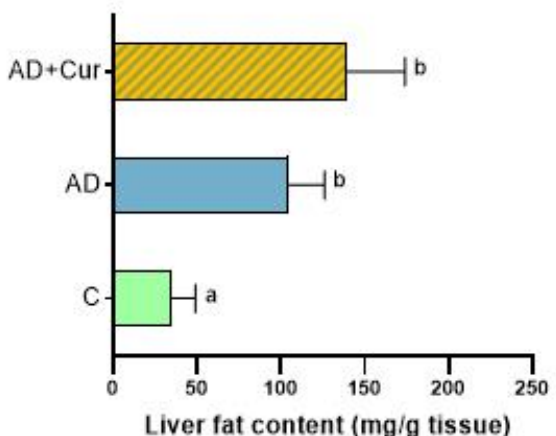

(C)

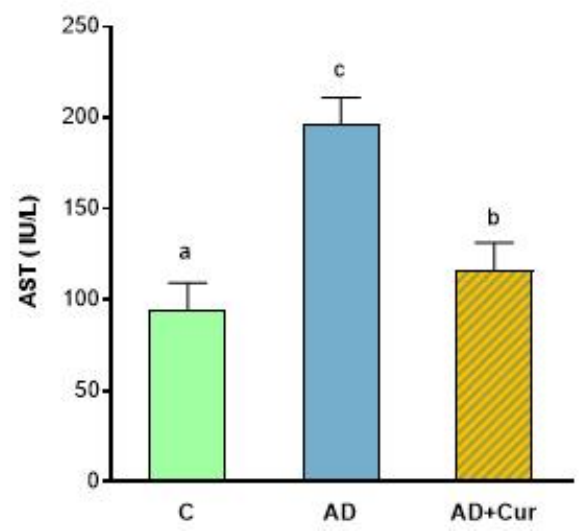

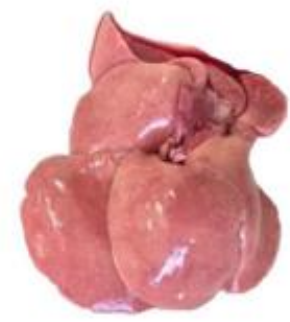

AD

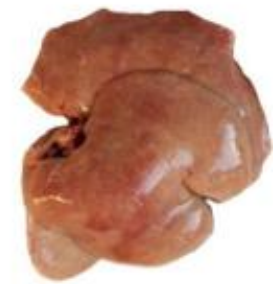

$\mathrm{AD}+\mathrm{Cur}$

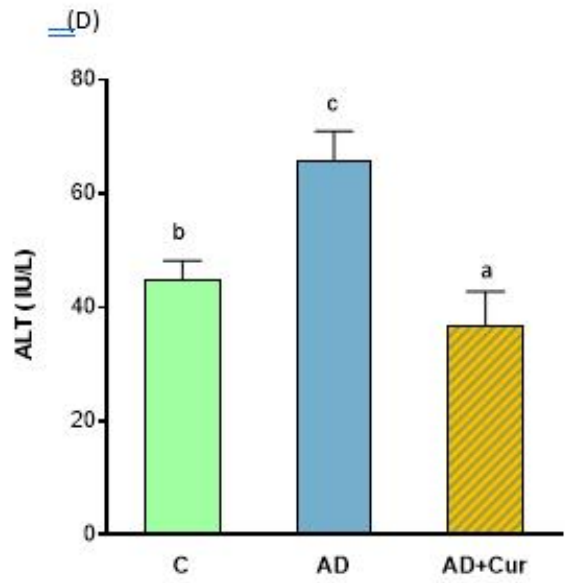

Figure 2. Supplementation with curcumin induces changes on hepatic morphology, liver fat content and enzymes of rats fed with an atherogenic diet. Rats were assigned into three groups: C, control group, was given access to a standard diet; AD group fed with atherogenic diet (saturated fatty acids and cholesterol diet) and $A D+C u r$ group (atherogenic diet and curcumin, saturated fatty acids and cholesterol diet plus oral curcumin). These diets were assigned for five weeks. Livers were removed and (A) hepatic morphology, (B) liver fat content (mg/g tissue), (C) aspartate aminotransferase (AST) activity and (D) alanine aminotransferase (ALT) activity were determined. (A): $A D$ induced hepatomegaly but morphologic differences among the three groups. Rats fed AD+Cur showed a liver between sharp red and dull pale. (B): hepatic steatosis was observed in rats fed AD or AD+Cur. (C) and (D): Cur induced a significant reduction in AST and ALT enzyme activity, respectively, provoked by AD diets. Data are mean values, with standard deviations. Data were analyzed by using ANOVA and Student-Newman-Keul's test $(P<0.05)$. Mean values with different letters indicate statistically significant differences between groups.

The histopathological evaluation of the liver sections performed after hematoxylin-eosin staining showed remarkable differences among groups (Figure 3). Feeding rats with $A D$ induced a higher steatosis and degeneration of the hepatocytes and the sinusoidal architecture than it did to those of the group C, fed a standard diet (Figure 3). In terms of hepatic steatosis, ADfed rats induced grade 3 lesions with macrovesicular vacuoles while $C$ rats were grade 0 (negative) for hepatic steatosis (Figure 3A). The marked increase in fat content displaced the nuclei of the affected cells to the periphery and induced microvesicular vacuoles and portal 
inflammation (mononuclear cells were typically predominant), and this condition affected the whole organ (Figure 3B). Although the supplementation with Cur did not show a decrease in liver fat content, histopathological differences were evident. Even though hepatocytes exhibited steatosis grade 3 and

microvesicular vacuoles, an apparent amelioration of inflammation was evident. The nuclei of these cells were not peripherally displaced in consistency with the absence of macrovesicular vacuoles. Then, Cur showed a hepatoprotective effect against inflammation $(P=0.002$, $\mathrm{Chi}^{2}$ test) and fewer lipid depositions (Figure 3C).

The DCFH-DA oxidation rate was measured in the liver isolated from $C$ and the animals treated with $A D$. The DCFH-DA oxidation was tested at different incubation times and it resulted to be linear with the experimental time, up to $15 \mathrm{~min}$ with high regression indexes under the conditions of the assay (Figure 4 inset).

In the homogenates from livers of the $A D$ supplemented animals, a significantly higher DCFH-DA oxidation rate was observed, as compared to $\mathrm{C}$ homogenates (Figure 4, 2.2-fold). However, in the homogenates from livers of rats that received $A D+C u r$, the values were significantly reduced by $25 \%$ comparing them with the $A D$ treated animals with Cur absence (Figure 4).

It is currently accepted that the DCFH-DA oxidation rate is dependent on a wide spectra of cellular oxidants [32]. The supplementation of the incubation medium with CAT (scavenger of $\mathrm{H}_{2} \mathrm{O}_{2}$ ), SOD (scavenger of $\mathrm{O}_{2}{ }^{-}$), DMSO (scavenger of ${ }^{\bullet} \mathrm{OH}$ ); GSH (general antioxidant) and deferoxamine (DF) (Fe chelator) were tested. The CAT, SOD and GSH addition to the assay conditions in $C$ liver homogenates produced significant reductions of the oxidation rate of the dye. However, DMSO and DF showed no differences over the basal results (Table 4). Under the experimental conditions used here, when rats were fed with $A D, C A T$ addition to the assay showed the highest inhibition of reactive species production. On the other hand, the homogenates of livers of animals receiving an $A D+C u r$ showed a different profile of the effects of the scavengers supplemented to the reaction medium. A significant inhibition of the DCFH-DA oxidation rate by either DF or SOD was determined with no significant changes by the supplementation with CAT, GSH or DMSO.

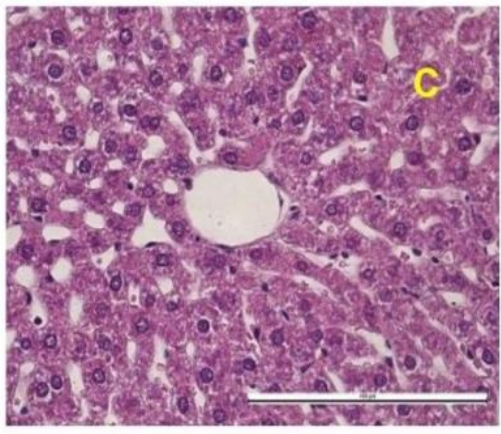

A

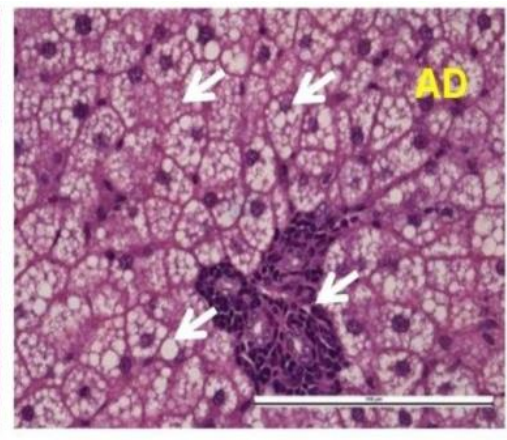

B

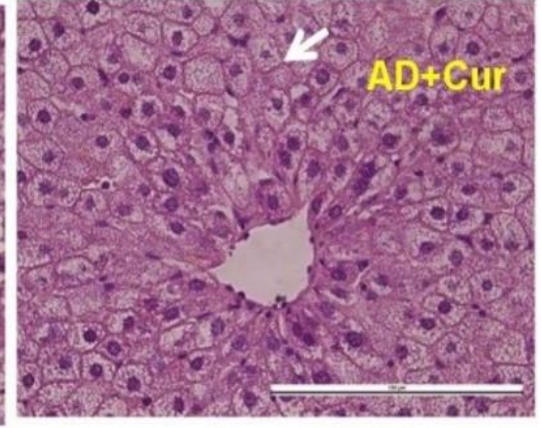

C

Figure 3. Supplementation $A D$ with curcumin diminished the hepatic lipid deposition. Rats were assigned into three groups: $C$, control group, was given access to a standard diet; $A D$ group fed with atherogenic diet (saturated fatty acids and cholesterol diet) and $A D+C u r$ group (atherogenic diet and curcumin, saturated fatty acids and cholesterol diet plus oral curcumin). These diets were assigned for five weeks. Livers were removed for histological evaluation. (A) Liver of $\mathrm{C}$ group showing normal hepatocytes and sinusoidal architecture; (B) Liver of AD showing steatosis grade 3 with macrovesicular and microvesicular steatosis with portal inflammation, and the nuclei were peripherally displaced (arrows); (C) Liver of $A D+C$ ur showing steatosis grade 3 with microvesicular steatosis with no inflammation. Cur shows a protective effect against inflammation. Samples stained with hematoxylin and eosin, 40x. 


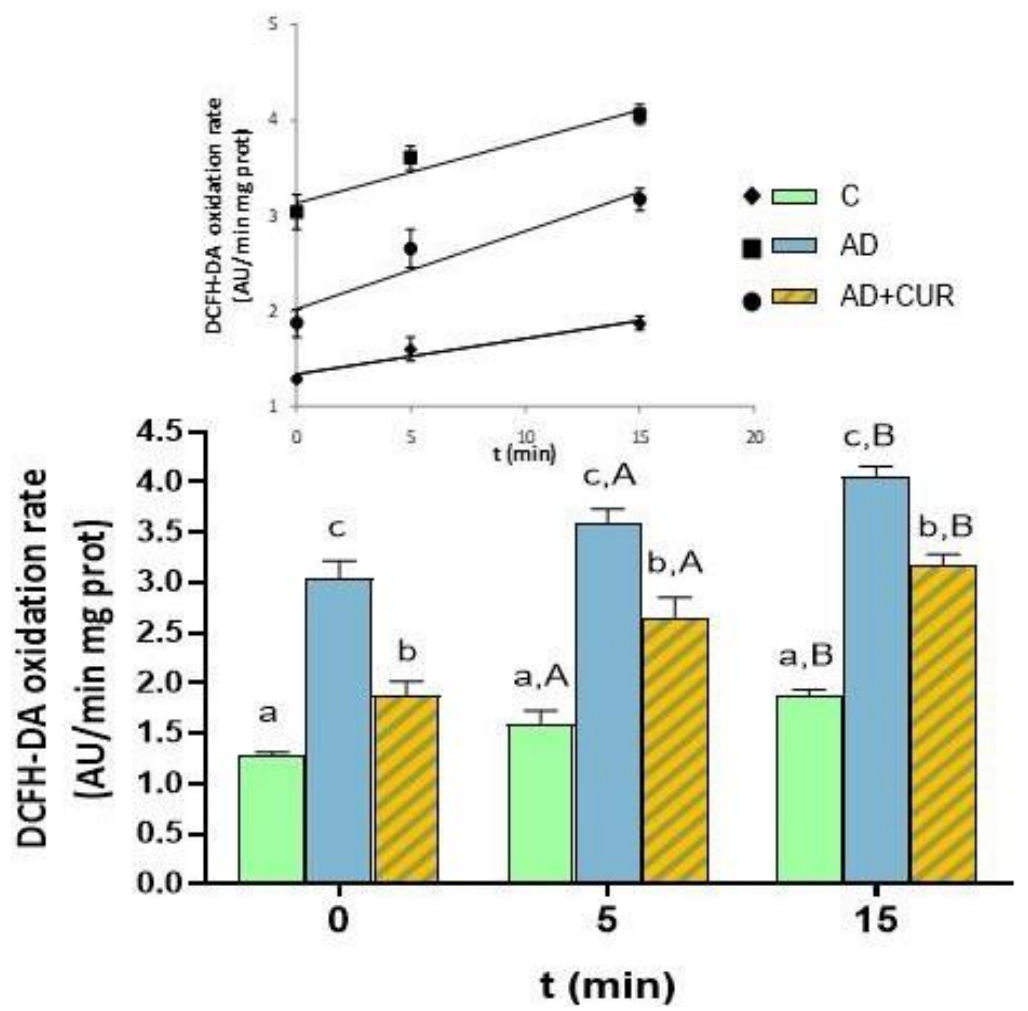

Figure 4. Supplementation with curcumin prevents hepatic oxidative stress of rats fed with an atherogenic diet. Rats were assigned into three groups: C, control group, was given access to a standard diet; AD group fed with atherogenic diet (saturated fatty acids and cholesterol diet) and AD+Cur group (atherogenic diet and curcumin, saturated fatty acids and cholesterol diet plus oral curcumin). These diets were assigned for five weeks. Livers were removed for oxidative stress assays. AD+Cur showed significant inhibition of the $2^{\prime}, 7^{\prime}$ dichlorofluorescein diacetate (DCFH-DA) oxidation rate by either deferoxamine (DF) or superoxide dismutase (SOD) with no significant changes on catalase (CAT), glutathione (GSH), or dimethyl sulfoxide (DMSO). Inset: linear regression curves for the reactive species generation during the DCFH-DA incubation period were for $\mathrm{C} y=0.04 \mathrm{x}+1.34$ ( $R 2=0.95)$; for $A D y=0.06 x+3.13(R 2=0.94)$; and for $A D+$ Cur $y=0.08 x+2.02(R 2=0.91)$. a,b,c Different letters indicate statistically significant differences between groups; ANOVA, (mean \pm $S D, p<0.0001)$. A, B. Different letters indicate statistically significant differences between the incubation time; ANOVA, ( $<<0.001)$.

Table 4. Effect of the exposure to scavengers on the DCFH-DA oxidation rate by liver homogenates from different rat diets. Data are expressed as $\mathrm{AU} / \mathrm{minmg}$ prot.

\begin{tabular}{lll|l} 
& $\mathrm{C}$ & $\mathrm{AD}$ & $\mathrm{AD}+\mathrm{Cur}$ \\
BASAL & $1.87 \pm 0.07 \mathrm{a}$ & $4.1 \pm 0.1 \mathrm{c}$ & $3.2 \pm 0.1 \mathrm{~b}$ \\
CAT & $0.8 \pm 0.1 \mathrm{~A} . \mathrm{a}(55 \%)$ & $0.8 \pm 0.1 \mathrm{~A} . \mathrm{a}(80 \%)$ & $3.0 \pm 0.1 \mathrm{~b}(6 \%)$ \\
SOD & $1.56 \pm 0.09 \mathrm{~A} . \mathrm{a} \mathrm{(17 \% )}$ & $3.6 \pm 0.1 \mathrm{~A} . \mathrm{c}(11 \%)$ & $2.5 \pm 0.3 \mathrm{~A} . \mathrm{b}(20 \%)$ \\
DMSO & $1.77 \pm 0.05 \mathrm{a}(5 \%)$ & $3.4 \pm 0.2 \mathrm{~A} . \mathrm{c}(17 \%)$ & $3.0 \pm 0.1 \mathrm{~b}(7 \%)$ \\
GSH & $1.56 \pm 0.06 \mathrm{~A} . \mathrm{a} \mathrm{(16 \% )}$ & $2.90 \pm 0.09 \mathrm{~A} . \mathrm{b}(28 \%)$ & $3.09 \pm 0.03 \mathrm{c}(3 \%)$ \\
DF & $1.9 \pm 0.1 \mathrm{a}(0 \%)$ & $2.7 \pm 0.3 \mathrm{~A} . \mathrm{b}(33 \%)$ & $1.5 \pm 0.2 \mathrm{~A} . \mathrm{a}(53 \%)$
\end{tabular}

C: control group (standard diet); AD: atherogenic diet group (saturated fatty acids and cholesterol diet); $A D+C u r:$ atherogenic diet and curcumin group (saturated fatty acids and cholesterol diet and oral curcumin). CAT: catalase; SOD: superoxide dismutase; DMSO: dimethylsulfoxide; GSH: glutathione; DF: deferoxamine. Percentage between brackets indicates the \% of inhibition of each scavenger compared to their basal group. Different lowercase letters indicate statistically significant differences between groups within each scavenger. ANOVA + Student-Newman-Keul's test $(p<0.001)$. A Significantly different to their own basal group. ANOVA + Dunnett's multiple comparisons test $(p<0.001)$. 


\section{DISCUSSION}

The present study demonstrated that supplementation with Cur to a high-fat high-cholesterol diet has a promising protective role acting as an anti-obesity agent and preventing liver injury; however, its cardioprotective effect could not be proved.

The consumption of a diet high in fat and cholesterol is associated with the development of certain metabolic disorders that promote chronic diseases.

To evaluate the benefits of Cur supplementation in preventing hepatic and metabolic alterations, the present study was performed in an experimental rat model of dietary induced hypercholesterolemia. This model appears to be more suitable for assessing liver alterations than other existing animal models, which require genetic deficiencies. Our results showed the capacity of oral Cur to reduce the total BW gain and body fat deposition, after 5 weeks of treatment. The lower BW gain observed could be attributed to Cur's increase of basal metabolism and energy expenditure [33]; further studies will be designed to elucidate these findings. Besides, Kim et al. [34] reported a significant decrease in BW gain and adiposity in rats fed a diet containing $10 \%$ of turmeric powder for 6 weeks. In concordance, as an antiobesity agent, Cur lowers body fat and reduces weight [35]. Indeed, Cur was reported to down-regulate the expression of genes involved in energy metabolism and lipid accumulation by decreasing the level of intracellular lipids [36]. In the adipose tissue, Cur suppresses the angiogenesis necessary for tissue growth [36]. In addition, Cur improves inflammation associated with obesity and may offer a cardioprotective effect for preventing hypercholesterolemia-induced

atherosclerosis [37].

In the present study, the highest increase in the amount of visceral fat was detected in rats fed with the $A D$, being this one of the risk factors for CVD. Nevertheless, the main predictors for CVD (non-HDL, HDL and total cholesterol) were not affected by Cur treatment. Meanwhile, Cur supplementation

ameliorated some consequences of consuming a high-fat high-cholesterol diet. However, the percentage of liver fat mass could not be reduced in Cur-treated rats. Five weeks exposition to Cur was not sufficient to improve the effects of hypercholesterolemia in the liver, highly associated with this detrimental AD. In fact, Cur proved to be a promising tool for improving liver function. However, three times higher values of hypercholesterolemia than normal prevented Cur from showing its beneficial effect on hepatic steatosis. These results may serve as a note of caution in the use of this type of diet. Further long-term studies including high fat and mild cholesterol diet should be performed.

As it was observed, the high-fat high-cholesterol feeding caused alterations in the lipid homeostasis evidenced by the increased T-C and non-HDL-C levels; but there was a lack of a lowering effect on serum T-C and non-HDL- C levels by Cur supplementation. Although hyperlipidemia was reported as one of the major risk factors for fatty liver [38], the serum TG levels were similar among groups. This may suggest that serum TG levels may not be an accurate indicator to evaluate fatty liver disorders in animals and, probably, in humans. It could be assumed that the hepatomegaly found in the $A D$ groups was possible due to the poor management of the dietary cholesterol metabolism [39]. Supplementation with Cur to AD did not show an improvement in the lipid profile. The incapacity to normalize the circulating levels T-C, non-HDL-C, and TG was not due to the low bioavailability of this compound, since Cur was coadministered in combination with an absorption enhancing adjuvant (piperine) [40]. However, plasma levels of Cur were not determined; the lack of this data might be a limitation that should be considered in further studies. These results are consistent with some meta- 
analyses which have found that Cur supplementation has apparently no effect on serum T-C, LDL-C, TG and HDL-C levels when considering heterogeneous populations [41]. Therefore, the proposed cardiovascular protective effects of Cur could be attributed to other mechanisms than lipid-lowering and HDL-C enhancing activities. Mechanisms such as mitigation of lipid peroxidation, platelet aggregation, endothelial dysfunction, and inflammation among others [41]. The results obtained are likely to be attributed to the trial duration, but not to an insufficient dose. According to the above, and taking into account that the metabolic rate in rats is higher than in humans, the amount of turmeric used for rats in the present study could be translated to an equivalent human dose of about $1 \mathrm{~g}$ of Cur per day, assuming that the average mass of a human is about $70 \mathrm{~kg}$ [42]. This amount of turmeric consumption is rational considering the capsules commercially available.

In contrast, other studies reported hypocholesterolemic effects of Cur in rats. Different mechanisms have been proposed for those hypolipidemic actions, including inhibition of intestinal cholesterol absorption, inhibition of hepatic lipid biosynthesis, stimulation of bile secretion and modulation of the expression and/or activity of lipoprotein receptors [40]. Rats fed with a $45 \%$ high-fat diet with or without Cur $(0.1 \% \mathrm{w} / \mathrm{w})$ for 8 weeks showed significantly decreased serum TG, T-C and LDL-C levels in comparison with controls. It was then suggested that Cur regulates the expression of genes involved in cholesterol homeostasis [43]. Even more, Cur was reported to transcriptionally inhibit 3-hydroxy-3-methylglutarylcoenzyme; a reductase (HMG-CoA) activity in a 18 week atherogenic model [44]. Some of the lipid-lowering effects of Cur in plasma might be responsible for its beneficial effects against several diseases in which hyperlipidemia plays important roles, such as inflammation, diabetes, obesity, and atherosclerosis [45].
In the present study, the $A D$ induced histopathological changes in the liver with an intense inflammatory infiltrate and steatosis which was observed by hematoxylin and eosin technique. In $A D$ and $A D+C u r$, some other histological techniques could be used to differentiate the steatosis grading more accurately; then, this limitation should be considered. Being the liver a major site for the synthesis, oxidation, metabolism, storage and distribution of lipids playing an essential role in regulating energy metabolism [46], the chronic consumption of this type of diets may lead to serious metabolic and liver alterations, such as fibrosis and cirrhosis [47]. Moreover, the additive cholesterol accretion in the liver brought steatohepatitis due to cholesterol-induced toxicity [48]. In the present experiments, Cur supplementation significantly decreased visceral fat and attenuated liver histopathology. The increase in the hepatic content of lipid was an important feature and showed the disturbance of lipid homeostasis due to a high-fat highcholesterol diet. A crucial fact is that even though the total amount of fat in the liver remained similar in rats fed with $A D$ or $A D+C u r$, it remains unclear how the contribution of each component -TG and cholesterolaffected the results. The morphological studies revealed a large difference in the hepatic appearance of each group of rats. Indeed, the $A D+C u r$ rats evidenced the existence of a different liver fat composition.

Hepatic enzymes such as ALT and AST are often used as markers of hepatic function and their serum increase is used as a marker of ongoing liver damage [49]. The results of the present study showed a significant increase in the serum activities of the AST and ALT in all rats consuming $A D$. The use of Cur evidenced its capacity to reduce AST and ALT as well as liver damage. These findings, confirmed by the histopathological observations of the liver, revealed that Cur could prevent liver damage and toxicity. The use of Cur evidenced its capacity to 
reduce AST and ALT as well as liver damage. Even though, specific studies were not performed to evaluate the correlation between oxidative stress development and the increase in these serum enzymatic activities in rats receiving the $A D$ diet, the measured enhancements on cellular radical generation lead to an increase in lipid peroxidation that favored the release of the liver enzymes to the blood. Thus Cur, by decreasing the oxidative stress, was able to prevent the leakage of the enzymes.

Feeding rats an AD induced hepatic steatosis due to a long-term accumulation of TG in the hepatocytes, often accompanied by increased oxidative stress. The excessive production of ROS and the antioxidant imbalance can provoke an acute inflammatory response, leading to progressive liver damage. ROS contribute to ischemia/regeneration, necrosis and apoptosis [14]. In this study, the oxidation rate of DCFH-DA was significantly increased in $A D$ rats as compared to $C$, suggesting that a high-fat high-cholesterol diet contributed to a cellular over production of total reactive species. $\mathrm{H}_{2} \mathrm{O}_{2}$, diffuses freely into the tissue increasing the oxidative stress and causes further oxidative damage. $\mathrm{H}_{2} \mathrm{O}_{2}$ is especially toxic through the Fenton reaction with $\mathrm{Fe}^{2+}$, where it becomes extremely reactive to ${ }^{\circ} \mathrm{OH}$, which causes severe damage to membranes, proteins, and DNA. Moreover, the excessive ROS production could overwhelm the scavengers leading to necrosis and apoptosis of hepatocytes [50]. Cur supplementation attenuated the oxidation rate of the dye induced by the $A D$, showing its role in the management and protection of oxidative damage associated with liver diseases. Cur was described to exert some hepatoprotective activity by preventing liver toxicity and reducing the activity of ALT and AST enzymes. These results were supported by the histopathological findings of the liver, observing an improvement in the morphological features of the hepatic tissue.

As shown here, when liver homogenates were exposed to scavengers, rats fed $A D$, showed the highest inhibition for CAT and were followed by DF, related to a $\mathrm{H}_{2} \mathrm{O}_{2}$ and $\bullet \mathrm{OH}$ production, respectively. In contrast, the Cur supplemented diet reduced the appearance of several reactive species but provoked the significant production of ${ }^{\bullet} \mathrm{OH}$. The lowest inhibition for CAT found in rats fed $A D+C u r$ could be caused by a lower production of $\mathrm{H}_{2} \mathrm{O}_{2}$ in the presence of Cur that prevented oxidative stress. Farzaei et al. [13] showed that Cur inhibits lipid peroxidation and neutralizes ROS.

\section{CONCLUSION}

Since findings suggest that oral supplementation with Cur improves some of the metabolic alterations associated with the consumption of an AD, Cur would act as an antiobesity agent reducing body fat and weight. It is evidenced here that Cur could not improve hypercholesterolemia and hepatic steatosis caused by DA; possibly due to the high concentration of cholesterol administered in the diet. Certainly, the amount of lipids supplied and the endogenous synthesis could exceed the hepatocyte's ability to manage its turnover rate thus establishing steatosis. However, the histological findings and the capacity of Cur to prevent liver inflammation in addition to its antioxidant effect, were evidenced in the reduction of ROS detected by the DCFH-DA assay. Lastly, the valuable use of Cur as a dietary supplement has a promising protective role against liver diseases although its cardioprotective capacity remains unproved. This study provides supporting evidence to confirm the beneficial effects of curcumin from the point of view of functional food science.

List of Abbreviations: AD: atherogenic diet, ALT: alanine aminotransferase, AST: aspartate aminotransferase, BW: body weight, C: Controls, Cur: curcumin, CAT: catalase, CVD: cardiovascular disease, DCFH-DA:

dichlorofluorescein diacetate, DF: deferoxamine, DMSO: dimethylsulfoxide, DRCD: diet-related chronic diseases, GSH: glutathione, HDL-C: high-density lipoproteincholesterol, HI: hepatic index, NAFLD: non-alcoholic fatty 
liver disease, non-HDL-C: non-HDL cholesterol, ROS:

reactive oxygen species, SOD: superoxide dismutase, T-C:

Total serum cholesterol, TG: triglycerides.

Competing interests: The authors declare no conflicts of interest. The authors alone are responsible for the content and writing of the article.

\section{Authors' contributions: MEA carried out the}

investigation, acquisition of data, analysis and

interpretation of data. PG helped design the study, analyzed the data and wrote the manuscript. CR, JC and CO processed samples, performed the lab determinations and analyzed the data. TS helped design the study, conducted the study, and analyzed the data. CM analyzed the histopathological data. VZ analyzed the serum lipids. SP designed the study, supervised the development of the study, analyzed the data, and wrote the manuscript. SMF analyzed the data and was a major contribution in manuscript preparation. EVM designed the study, supervised the development of the study, analyzed the data, and wrote the manuscript. All authors read and approved the final manuscript.

Acknowledgment: We thank Ricardo Orzuza for technical assistance and care of experimental animals.

Funding: This study was supported by the University of Buenos Aires grants 20020150200013BA, 20020170100138BA, 20020170200055BA and 20020170100199BA and the National Council for Science and Technology (CONICET, PIP 11220170100539CO) Argentina

\section{REFERENCES}

1. Amor S, González-Hedström D, Martín-Carro B, InarejosGarcía AM, Almodóvar P, Prodanov M, García-Villalón AL, García MG. Beneficial effects of an aged black garlic extract in the metabolic and vascular alterations induced by a high fat/sucrose diet in male rats. Nutrients 2019, 11:153. https://doi.org/10.3390/nu11010153
2. Kyrou I, Randeva HS, Tsigos C, Kaltsas G, Weickert MO. Clinical Problems Caused by Obesity. In: Feingold KR, Anawalt B, Boyce A, et al., editors. Endotext [Internet]. South Dartmouth (MA): MDText.com, Inc.; 2000-. [Updated 2018 Jan 11]. https://www.ncbi.nlm.nih.gov/books/NBK278973/

3. Fardet A, Boirie Y.Associations between food and beverage groups and major diet-related chronic diseases: an exhaustive review of pooled/meta-analyses and systematic reviews. Nutrition Reviews 2014, 72:741-762.

https://doi.org/10.1111/nure.12153

4. Health in the Americas. Health status of the population. Noncommunicable disease prevention and control. PAHO [https://www.paho.org/salud-en-las-americas-2017/rononcommunicable.html] Retrieved October 1, 2021.

5. Recena Aydos L, Aparecida do Amaral L, Serafim de Souza R, Jacobowski AC, Freitas dos Santos E, Rodrigues Macedo ML. Nonalcoholic Fatty Liver Disease Induced by High-Fat Diet in C57bl/6 Models. Nutrients 2019, 11:3067. https://doi.org/10.3390/nu11123067

6. Araújo AR, Rosso N, Bedogni G, Tiribelli C, Bellentani S. Global epidemiology of non-alcoholic fatty liver disease/nonalcoholic steatohepatitis: What we need in the future. Liver International 2018, 38:47-51.

https://doi.org/10.1111/liv. 13643

7. Younossi ZM, Koenig AB, Abdelatif D, Fazel Y, Henry L, Wymer M. Global epidemiology of nonalcoholic fatty liver diseaseMeta-analytic assessment of prevalence, incidence, and outcomes. Hepatology 2016, 64:73-84. https://doi.org/10.1002/hep.28431

8. Marchisello S, Di Pino A, Scicali R, Urbano F, Piro S, Purrello F, Rabuazzo AM. Pathophysiological, molecular and therapeutic issues of nonalcoholic fatty liver disease: An overview. International Journal of Molecular Sciences 2019, 20:1-33. https://doi.org/10.3390/ijms20081948

9. Jones DP Redefining oxidative stress. Antioxidants and Redox Signaling. 2006, 8:1865-1879.

https://doi.org/10.1089/ars.2006.8.1865

10. Masarone M, Rosato V, Dallio M, Gravina AG, Aglitti A, Loguercio C, Federico A, Persico M. Role of oxidative stress in pathophysiology of nonalcoholic fatty liver disease. Oxidative Medicine and Cellular Longevity 2018, 2018:1-15. https://doi.org/10.1155/2018/9547613

11. Vial G, Dubouchaud H, Couturier K, Cottet-Rousselle C, Taleux N, Athias A, Galinier A, Casteilla L, Leverve XM. Effects of a high-fat diet on energy metabolism and ROS production in rat liver. Journal of Hepatology 2011, 54:348-356. https://doi.org/10.1016/j.jhep.2010.06.044 
12. Cichoz-Lach $\mathrm{H}$, Michalak A. Oxidative stress as a crucial factor in liver diseases. World Journal of Gastroenterology 2014, 20:8082-8091.

13. Farzaei $\mathrm{MH}$, Zobeiri M, Parvizi F, El-Senduny FF, Marmouzi I, Coy-Barrera E, Naseri R, Nabavi SM, Rahimi R, Abdollahi M. Curcumin in liver diseases: A systematic review of the cellular mechanisms of oxidative stress and clinical perspective. Nutrients 2018, 10:1-18. https://doi.org/10.3390/nu10070855

14. Vera-Ramirez L, Pérez-Lopez P, Varela-Lopez A, RamirezTortosa M, Battino M, Quiles JL. Curcumin and liver disease. BioFactors 2013, 39:88-100. https://doi.org/10.1002/biof.1057

15. Hewlings S, Kalman D. Curcumin: A Review of Its' Effects on Human Health. Foods 2017, 6:92. https://doi.org/10.3390/foods6100092

16. Srivastava A, Gupta RC, Doss RB, Lall R. Trace Minerals, Vitamins and Nutraceuticals in Prevention and Treatment of COVID-19. Journal of Dietary Supplements 2021, 1-35. https://doi.org/10.1080/19390211.2021.1890662

17. Jayarathne S, Koboziev I, Park OH, Oldewage-Theron W, Shen CL, Moustaid-Moussa N. Anti-Inflammatory and Anti-Obesity Properties of Food Bioactive Components: Effects on Adipose Tissue. Preventive Nutrition and Food Science 2017, 22:251262.

18. Council. NR, Animals. C for the $U$ of the $G$ for the $C$, Laboratory $U$ of, Research. I for LA.Guide for the Care and Use of Laboratory. National Research Council (US) Guide for the Care and Use of Laboratory Animals 2011, 220. https://doi.org/10.17226/12910

19. Ganiger S, Malleshappa HN, Krishnappa H, Rajashekhar G, Ramakrishna Rao V, Sullivan F. A two generation reproductive toxicity study with curcumin, turmeric yellow, in Wistar rats. Food and Chemical Toxicology 2007, 45:64-69. https://doi.org/10.1016/i.fct.2006.07.016

20. Panahi Y, Alishiri GH, Parvin S, Sahebkar A. Mitigation of Systemic Oxidative Stress by Curcuminoids in Osteoarthritis: Results of a Randomized Controlled Trial. Journal of Dietary Supplements 2016, 13:209-220. https://doi.org/10.3109/19390211.2015.1008611

21. Kale VP, Joshi GS, Gohil PB, Jain MR. Effect of fasting duration on clinical pathology results in Wistar rats. Veterinary Clinical Pathology 2009, 38:361-366. https://doi.org/10.1111/j.1939-165X.2009.00143.x

22. Qin $Y$, Tian Y. Preventive effects of chronic exogenous growth hormone levels on diet-induced hepatic steatosis in rats.
Lipids in Health and Disease 2010, 9:78.

https://doi.org/10.1186/1476-511X-9-78

23. Giordano A, Frontini A, Cinti S. Convertible visceral fat as a therapeutic target to curb obesity. Nature Reviews Drug Discovery 2016, 15:405-424. https://doi.org/10.1038/nrd.2016.31

24. Folch J, Lees M, Sloane Stanley GH. A simple method for the isolation and purification of total lipides from animal tissues. The Journal of biological chemistry 1957, 226:497-509. https://doi.org/10.1016/s0021-9258 (18)64849-5

25. Kleiner DE, Brunt EM, Van Natta M, Behling C, Contos MJ, Cummings OW, Ferrell LD, Liu Y-C, Torbenson MS, UnalpArida A, Yeh M, McCullough AJ, Sanyal AJ, Nonalcoholic Steatohepatitis Clinical Research Network. Design and validation of a histological scoring system for nonalcoholic fatty liver disease. Hepatology 2005, 41:1313-1321. https://doi.org/10.1002/hep.20701

26. Lucero D, Olano C, Bursztyn M, Morales C, Stranges A, Friedman S, Macri E V., Schreier L, Zago V. Supplementation with $n-3, n-6, n-9$ fatty acids in an insulin-resistance animal model: Does it improve VLDL quality? Food and Function 2017, 8:2053-2061. https://doi.org/10.1039/c7fo00252a

27. Alsina E, Macri E V., Lifshitz F, Bozzini C, Rodriguez PN, Boyer PM, Friedman SM. Efficacy of phytosterols and fish-oil supplemented high-oleic-sunflower oil rich diets in hypercholesterolemic growing rats. International Journal of Food Sciences and Nutrition 2016, 67:441-453. https://doi.org/10.3109/09637486.2016.1161010

28. Gallagher EP, Canada AT, Di Giulio RT. The protective role of glutathione in chlorothalonil-induced stoxicity to channel catfish. Aquatic Toxicology 1992, 23:155-168. https://doi.org/10.1016/0166-445X(92)90049-S

29. Amado LL, Garcia ML, Ramos PB, Freitas RF, Zafalon B, Ferreira JLR, Yunes JS, Monserrat JM. A method to measure total antioxidant capacity against peroxyl radicals in aquatic organisms: Application to evaluate microcystins toxicity. Science of the Total Environment 2009, 407:2115-2123. https://doi.org/10.1016/j.scitotenv.2008.11.038

30. Viarengo A, Burlando B, Cavaletto M, Marchi B, Ponzano E, Blasco J. Role of metallothionein against oxidative stress in the mussel Mytilus galloprovincialis. American Journal of Physiology - Regulatory Integrative and Comparative Physiology 1999, 277:R1612-R1619. https://doi.org/10.1152/ajpregu.1999.277.6.R1612

31. Lowry $\mathrm{OH}$, Rosebrough NJ, Farr AL, Randall RJ. Protein measurement with the Folin phenol reagent. The Journal of biological chemistry 1951, 193:265-75. 
32. Tarpey MM, Wink DA, Grisham MB. Methods for detection of reactive metabolites of oxygen and nitrogen: In vitro and in vivo considerations. American Journal of Physiology Regulatory Integrative and Comparative Physiology 2004, 286. https://doi.org/10.1152/ajpregu.00361.2003

33. Hasan ST, Zingg JM, Kwan P, Noble T, Smith D, Meydani M. Curcumin modulation of high fat diet-induced atherosclerosis and steatohepatosis in LDL receptor deficient mice. Atherosclerosis 2014, 232:40-51. https://doi.org/10.1016/j.atherosclerosis.2013.10.016

34. Kim JG, Mandal PK, Choi KD, Pyun CW, Hong GE, Lee CH. Beneficial dietary effect of turmeric and sulphur on weight gain, fat deposition and lipid profile of serum and liver in rats. Journal of Food Science and Technology 2014, 51:774-779. https://doi.org/10.1007/s13197-011-0569-8

35. González-Castejón M, Rodriguez-Casado A. Dietary phytochemicals and their potential effects on obesity: A review. Pharmacological Research 2011, 64:438-455. https://doi.org/10.1016/j.phrs.2011.07.004

36. Rahman A. Studies in Natural Products Chemistry. Studies in Natural Products Chemistry 2003, 29:ii. https://doi.org/10.1016/S1572-5995 (03)80020-0

37. Mauren FM, Yanti, Lay BW. Efficacy of oral curcuminoid fraction from curcuma xanthorrhiza and curcuminoid cider in high-cholesterol fed rats. Pharmacognosy Research 2016, 8:153-159. https://doi.org/10.4103/0974-8490.181468

38. Mahamid M, Khoury T, Mahamid B, Sbeit W, Mari A, Nseir W. The interplay between abdominal aortic aneurysm, metabolic syndrome and fatty liver disease: A retrospective case-cont. Diabetes, Metabolic Syndrome and Obesity: Targets and Therapy 2019, 12:1743-1749. https://doi.org/10.2147/DMSO.S205568

39. Lee S, Lee MS, Chang E, Lee Y, Lee J, Kim J, Kim CT, Kim IH, Kim $\mathrm{Y}$. Mulberry fruit extract promotes serum hdl-cholesterol levels and suppresses hepatic microrna-33 expression in rats fed high cholesterol/cholic acid diet. Nutrients 2020, 12:1-14. https://doi.org/10.3390/nu12051499

40. Moohebati M, Yazdandoust S, Sahebkar A, Mazidi M, SharghiShahri Z, Ferns G, Ghayour-Mobarhan M. Investigation of the effect of short-term supplementation with curcuminoids on circulating small dense low-density lipoprotein concentrations in obese dyslipidemic subjects: A randomized double-blind placebo-controlled cross-over trial. ARYA atherosclerosis 2014, 10:280-6.
41. Sahebkar A. A systematic review and meta-analysis of randomized controlled trials investigating the effects of curcumin on blood lipid levels. Clinical Nutrition 2014, 33:406-414. https://doi.org/10.1016/j.clnu.2013.09.012

42. Nair A, Jacob S. A simple practice guide for dose conversion between animals and human. Journal of Basic and Clinical Pharmacy 2016, 7:27.

https://doi.org/10.4103/0976-0105.177703

43. Kim M, Kim Y.Hypocholesterolemic effects of curcumin via upregulation of cholesterol 7a-hydroxylase in rats fed a high fat diet. Nutrition Research and Practice 2010, 4:191. https://doi.org/10.4162/nrp.2010.4.3.191

44. Shin S-K, Ha T-Y, McGregor RA, Choi M-S. Long-term curcumin administration protects against atherosclerosis via hepatic regulation of lipoprotein cholesterol metabolism. Molecular Nutrition and Food Research 2011, 55:1829-1840. https://doi.org/10.1002/mnfr.201100440

45. Zingg JM, Hasan ST, Meydani M. Molecular mechanisms of hypolipidemic effects of curcumin. BioFactors 2013, 39:101121. https://doi.org/10.1002/biof.1072

46. Rui L. Energy Metabolism in the Liver. In: Comprehensive Physiology. John Wiley and Sons, Inc., Hoboken, NJ, USA, 2014, 177-197. https://doi.org/10.1002/cphy.c130024

47. Barroso MV, Graça-Reis A, Cattani-Cavalieri I, Gitirana LB, Valenca SS, Lanzetti M. Mate tea reduces high fat dietinduced liver and metabolic disorders in mice. Biomedicine and Pharmacotherapy 2019,109: 1547-1555.

https://doi.org/10.1016/i.biopha.2018.11.007

48. Matsuzawa N, Takamura T, Kurita S, Misu H, Ota T, Ando H, Yokoyama M, Honda M, Zen Y, Nakanuma Y, Miyamoto KI, Kaneko S. Lipid-induced oxidative stress causes steatohepatitis in mice fed an atherogenic diet. Hepatology 2007, 46:1392-1403. https://doi.org/10.1002/hep.21874

49. White CM, Lee JY. The impact of turmeric or its curcumin extract on nonalcoholic fatty liver disease: A systematic review of clinical trials. Pharmacy Practice 2019, 17:1350. https://doi.org/10.18549/PharmPract.2019.1.1350

50. Redza-Dutordoir M, Averill-Bates DA. Activation of apoptosis signalling pathways by reactive oxygen species. Biochimica et Biophysica Acta (BBA) - Molecular Cell Research 2016, 1863:2977-2992. https://doi.org/10.1016/j.bbamcr.2016.09.012 